\title{
The trim coils for the Wendelstein 7-X magnet system
}

\author{
Thomas Rummel, Konrad Riße, Johann Kißlinger, Matthias Köppen, Frank Füllenbach, Hutch \\ Neilson, Tom Brown, and Subrahmanya Ramakrishnan
}

\begin{abstract}
The magnet system of the fusion experiment Wendelstein 7-X (W7-X) consists of superconducting as well as normal conducting coils. 50 non planar superconducting coils are forming the main field, 20 planar superconducting coils allow varying the shape of the plasma. Inside of the plasma vessel 10 normal conducting control coils will be placed to modify the strike points of the plasma at the divertor. In addition a set of five normal conducting trim coils has been designed to allow the correction of error fields and to increase the experimental flexibility. The coils will be placed at the outer surface of the outer vessel of W7-X. Four out of five coils have identical size and shape. They have dimensions of 3.5x3.3 meters with 48 turns and will be operated with currents of up to $1.8 \mathrm{kA}$. The other coil type has a smaller size of $2.8 \times 2.2$ meters, but a higher number of turns and a higher operating current of $1.95 \mathrm{kA}$. Both types of trim coils will be made of square copper hollow profile with an integrated cooling channel. Five independent power supplies will be used to energize the coils. The present concept is based on four-quadrant power supplies. The control system will allow the local control as well as the remote control of the five power supplies from an external control room.
\end{abstract}

Index Terms-Wendelstein $7-\mathrm{X}$, fusion reactors, normal conducting coils, power supplies

\section{INTRODUCTION}

$\mathrm{T}$ HE magnet system of the fusion experiment Wendelstein 7-X (W7-X) consists of superconducting as well as normal conducting coils. 50 non planar superconducting coils are forming the fivefold symmetrical main field, 20 planar superconducting coils allow varying the shape of the plasma. Inside of the plasma vessel 10 normal conducting control coils will be placed to modify the strike points of the plasma at the divertor.

In addition a set of five normal conducting trim coils has been designed to allow the correction of error fields and to increase the experimental flexibility by providing a means to balance the divertor heat loads among the five field periods. The coils will be placed at the outer surface of the outer vessel of W7-X and directly in the middle of each of the five $\mathrm{W} 7-\mathrm{X}$ main

Manuscript received 12 September 2011. This work was supported in part by the U.S. Department of Energy.

Th. Rummel, K. Riße, J. Kißlinger, M. Köppen and F. Füllenbach are with the Max-Planck-Institut für Plasmaphysik, 17491 Greifswald, Germany, (corresponding author phone: +49 383488 2778; fax: +49 383488 2709; email: thomas.rummel@ipp.mpg.de).

H. Neilson, T. Brown and S. Ramakrishnan are with Princeton Plasma Physics Laboratory, Princeton, NJ 08543 USA, (e-mail: hneilson@pppl.gov). modules.

The trim coil project will be handled in close collaboration between the Princeton Plasma Physics Laboratory (PPPL) and the Max-Planck-Institute for Plasma Physics Greifswald (IPP). The coils will be manufactured by PPPL and the support elements and the assembly will be provided by IPP.

\section{MOTIVATION FOR USING TRIM COILS}

During fabrication and assembly of the W7-X coils outmost care is taken to keep tolerances small. However, even these small deviations from the ideal shape and position of the coils along with other influences like the presence of minor quantities of ferromagnetic material or the unavoidable deformation of the support structures during operation of the machine will result in magnetic field errors. Especially errors which break the fivefold symmetry of the W7-X lead to additional magnetic islands and asymmetric loads on the divertor [1]. Fig. 1 shows an example of magnetic islands for a relatively small asymmetric error. In this case, just due to this changed, asymmetric shape of the magnetic islands only two out of the ten W7-X targets modules would take almost the complete load, resulting in an asymmetric power distribution which needs to be avoided.

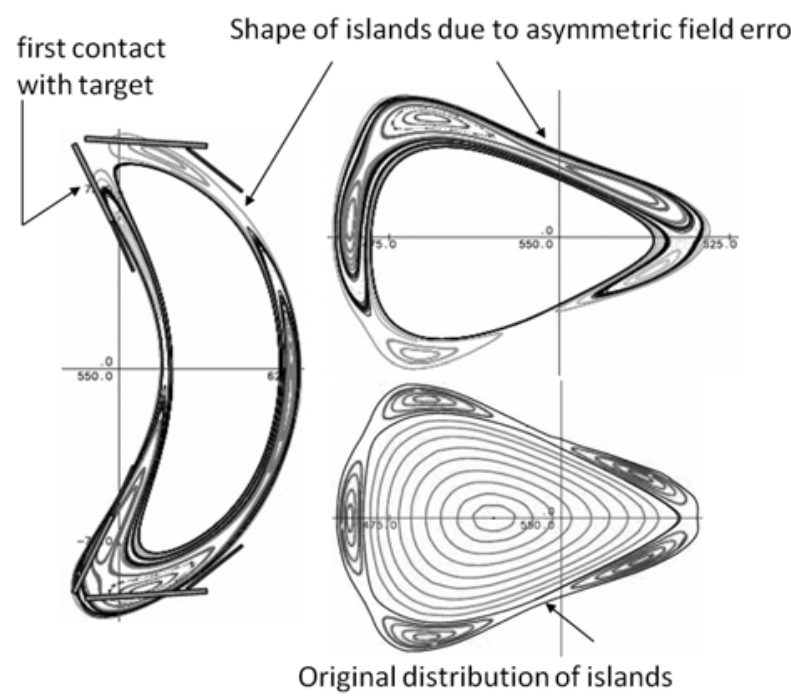

Fig. 1. Magnetic islands and contacts with targets for a small asymmetric error (module inclination of $0.1^{\circ}$ ).

In W7-X one trim coil per module will be installed and supplied with an individual current. These trim coils can be used to provide strong individual field components which, together with the 10 smaller control coils in the plasma vessel, 
facilitate the compensation of field errors - not per superconducting coil, but per module. It may be noted that the use of the trim coils is not only limited to the correction of field errors. They also increase the experimental flexibility by allowing the investigation of the effects of magnetic islands on the magnetic confinement and by offering an option to influence the helical plasma axis.

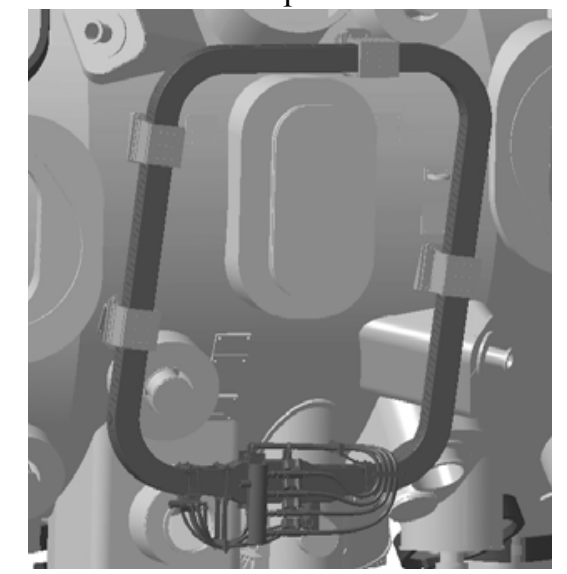

Fig. 2. Trim coil type B with four supports and lead area at the bottom.

\section{TRIM COIL DESIGN}

\section{A. Basic design criteria}

The main magnet system of W7-X consists of non planar, planar and control coils that are fully symmetric. But the arrangement of ports at the outer side along the external side of the outer vessel differs from module to module. Therefore the trim coils overall shape was designed in a way to avoid areas where ports with diagnostics, supply lines or plasma heating systems are placed. A second design criteria was the requirement that the coil manufacturing should allow winding under tension to keep the costs down. Also for economical reason it was decided to design the planar coils and hence they do not follow the shape of the outer vessel. Due to space restrictions only four out of five coils have identical size and shape (type A). They have overall dimensions of $3.5 \times 3.3$ meters with 48 turns and will be operated with currents of up to $+/-1.8 \mathrm{kA}$. Due to limited space the trim coil type B, see fig. 2 , is smaller (2.8×2.2 meters) than the type A coils, which are identical to each other. To achieve the same effect on the plasma, the smaller trim B coil consists of more turns -72 instead of 48 - and is designed to carry a higher current- 1.95 $\mathrm{kA}$ instead of $1.8 \mathrm{kA}$. It is also located slightly closer to the superconducting coils and the confined plasma. Table 1 lists the main parameter of the coils.

Similar to all the other coils in W7-X, the trim coils had to be designed for steady state operation.

\section{B. Conductor}

The trim coils are normal conducting coils using oxygen free copper according to ASTM B188 UNS C10200. The conductivity shall be $100 \%$ minimum IACS (min.) at 20 degrees $\mathrm{C}$. The conductor is a square copper hollow profile with outer dimensions of $16.26 \times 16.26 \mathrm{~mm}$ with an integrated cooling channel of $10.16 \mathrm{~mm}$. During discussions with prospective manufacturers it turned out that the relatively small design tolerances in terms of outer dimension and cooling channel diameter cannot be met and had to be enlarged to $+/-0.5 \mathrm{~mm}$ for both, the outer dimensions and the cooling channel diameter. In order to address this situation a selection process will be incorporated in the manufacturing procedure to select conductors with nearly equal dimension to be integrated into the same coil. In general the conductors with the largest outer dimensions should not be used in the type B coil due to space restrictions.

\section{TABLE 1 MAIN PARAMETERS OF THE TRIM COILS}

\begin{tabular}{|c|c|c|}
\hline Designation & Type A Coil & Type B Coil \\
\hline No. of turns. & 48 & 72 \\
\hline Inductance & $19 \mathrm{mH}$ & $26 \mathrm{mH}$ \\
\hline Resistance@20C & $53 \mathrm{~m} \Omega$ & $59 \mathrm{~m} \Omega$ \\
\hline Power loss & $200 \mathrm{~kW}$ & $260 \mathrm{~kW}$ \\
\hline Operation current & $+/-1800 \mathrm{~A}$ & $+/-1950 \mathrm{~A}$ \\
\hline Water pressure drop & 3 bar & 5 bar \\
\hline Conductor cross section & \multicolumn{2}{|c|}{$180 \mathrm{~mm}^{2}$} \\
\hline Test voltage to ground & \multicolumn{2}{|c|}{$1 \mathrm{kV}$} \\
\hline $\begin{array}{l}\text { Insulation resistance to } \\
\text { ground }\end{array}$ & \multicolumn{2}{|c|}{$1 \mathrm{M} \Omega$} \\
\hline $\begin{array}{l}\text { No. of load cycles (design } \\
\text { value) }\end{array}$ & \multicolumn{2}{|c|}{60,000} \\
\hline
\end{tabular}

For the manufacturing of the coils a total 40 lengths each 90 meters long are necessary. To allow the above indicated selection and to have some spare lengths a total of 50 lengths have been ordered.

Planned conductor tests include geometrical measurements, and tests of the mechanical parameters of the conductor material such as yield and ultimate strengths.

\section{Winding pack}

Both types of trim coils will be made of eight pancakes. The type A coil will have 6 turns per pancake leading to 48 turns and the type B coil will have 9 turns per pancake and 72 turns in total. A vacuum pressure impregnated insulation based on glass tapes and epoxy resin provides the electrical insulation as well as the mechanical stiffness of the winding pack. The turn to turn insulation has a thickness of $0.4 \mathrm{~mm}$, the layer to layer insulation will have a thickness of $1.0 \mathrm{~mm}$ and for the insulation to ground a thickness of $4 \mathrm{~mm}$ is foreseen. The insulation will be done with Boron free S-2 glass tapes and mats, vacuum pressure impregnated with epoxy and cyanate ester resin. In addition a cyanate ester based primer will be used for improved bonding of resin/blend to the copper.

The cooling of the coils will be provided by de-ionized water with a maximum conductivity of $5 \mu \mathrm{S} / \mathrm{m}$. Since the coils will be wound in double pancakes, the cooling scheme is based on single pancake cooling to keep the pressure drop per circuit low in order to meet to the W7-X overall cooling water parameters. The flow rates are expected to be 4.3 and 5.6 
cubic meter per hours. This will lead to an average temperature rise of $30 \mathrm{~K}$ over the coil. The whole design provides for steady state operation.

The maximum operation temperature will be observed by thermocouples and voltage taps between the water inlets and outlets.

\section{ELECTROMAGNETIC ANALYSIS OF THE TRIM COILS}

The described trim coils with the designed ampere turns create large field components. Before adding them to the project, all their influences on the rest of the machine, along with resultant influences of the W7-X on them had to be analyzed. Therefore the following analyses have been performed:

\section{A. Stray fields}

For safety reasons, but also in order to estimate the possible necessity of local magnetic shielding, stray fields for the nine reference magnetic field configurations of the $\mathrm{W} 7-\mathrm{X}$ have been calculated. However, due to the strong field of the W7-X superconducting coils the contribution of the trim coils to stray fields is relatively small.

\section{B. Forces acting on the trim coils}

During operation electromagnetic forces will act on the trim coils. These forces result from a superposition of different field components. The self field of a trim coil will lead to in plane hoop forces, trying to widen the coil. These forces are strongest close to bends in the winding pack, where also the local magnetic self field reaches maximum values.

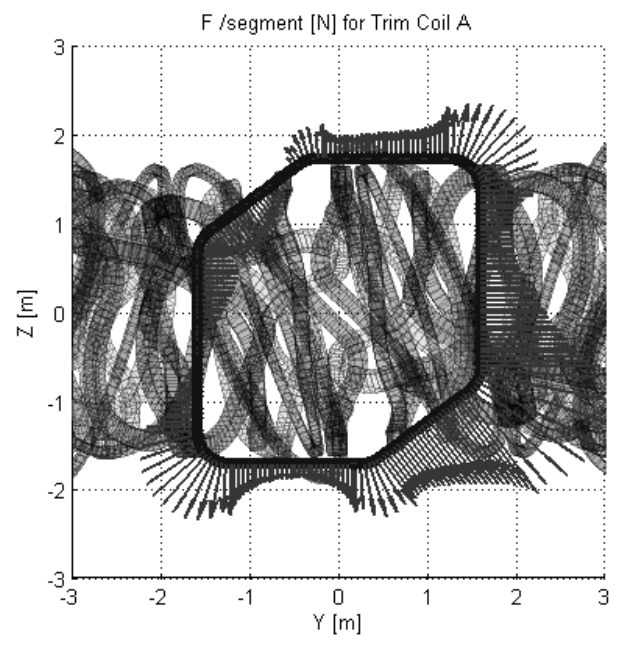

Fig. 3. Force vectors (arrows) on segments of a trim coil type A seen together with the nearby W7-X superconducting coils in the background

The external field coming from the W7-X superconducting coils is not homogeneous. It can therefore lead to non homogeneous and out of plane forces that will depend on the current distribution over the superconducting coil groups. The largest forces can be expected for scenarios with strong currents in the coils closest to the trim coils. The High Iota configuration of W7-X features relatively strong currents in all the superconducting coils, but especially the maximum currents in the planar coils, which are closest to the trim coils. Fig. 3 shows an example of the trim coil in module 1 in High
Iota. While the self field of the trim coil would always lead to radially outward forces, the force direction is actually turned around by almost $180^{\circ}$ in the upper left hand corner, just due to the strong external field from nearby superconducting coils. The opposite effect can be observed in the lower right hand corner, where the forces resulting from the external field just add to the already strong self field forces.

Fig. 4 shows the resulting distribution of force densities along the circumference of the winding pack of the trim coils in modules 1,3,4, and 5 in self field (SF) and also in all the nine reference magnetic field configurations of W7-X superimposed on the self field.

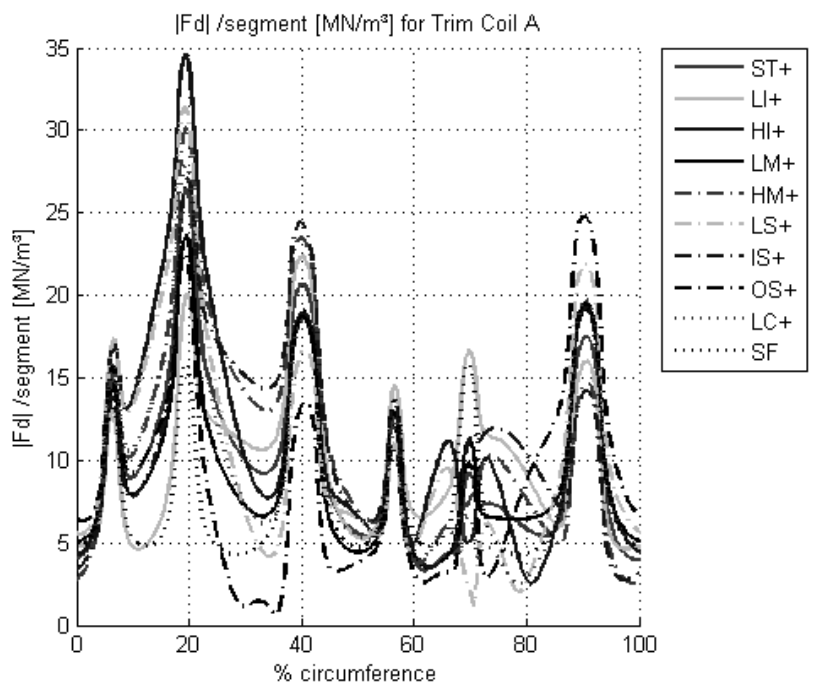

Fig. 4. Force density distribution on trim coil in self field (SF) and various external fields + self field

The resulting fields were also used to estimate forces acting on the power supply cables connected to the coils, which are, however, relatively small.

\section{Fast discharge of W7-X}

In case of a quench, all superconducting coils of the W7-X will be quickly discharged at the same time. The changing field will induce voltages in all surrounding geometries. In case of the trim coils this could lead to additional coil currents which would have to be considered.

However, when calculating the inductance matrix for W7 X including the trim coils, one finds mutual inductances between the trim coils and the superconducting coils, which are very close to zero. In fact only the trim coil in module 2 (type B) shows a noticeable magnetic coupling. This is due to the position of the trim coils in stellarator symmetry, with the symmetry axis through the centre of the trim coil and a symmetric distribution of the coil area around this axis. Any change in the magnetic flux density from the W7-X perpendicular to this area in one half of the trim coil is effectively canceled out by an equally large change in the opposite direction in the stellarator symmetric other half of the coil area, leading to an overall time derivative of the magnetic flux equal to zero. Without a resulting time derivative of the enclosed magnetic flux no additional voltage is induced. 


\section{POWER SUPPLIES FOR THE TRIM COILS}

It was decided to design five independent power supplies to energize the coils. This provides the maximum flexibility for the operation.

The power supply system comprises the power supply units, Disconnect \& Ground Switches, a low voltage distribution, a cooling plant and a control system including all services and supports. PPPL will provide to W7-X the five power supplies and the local controls. IPP is responsible for the central control, the central cooling water unit, all cabling and raceways to the coils and the assembly.

In the following sections the main parts of the trim coil power supply package will be described:

\section{A. Power converter}

Each trim coil will be fed by a dedicated power supply unit connected to the $0,4 \mathrm{kV}$ grid. The electrical parameters from trim coil type B differ significantly from the ones of the type A coils. The requirements for the power supply unit are therefore tailored to this particular need (table 2). Nevertheless there could be an option based upon technical and economical constraints to standardize the five power supply units. Technical and economical conditions will be taken into account.

\section{TABLE 2 MAIN PARAMETERS OF THE POWER SUPPLIES}

\begin{tabular}{|c|c|c|}
\hline Designation & Type A Coil & Type B Coil \\
\hline Output voltage max. & $180 \mathrm{~V}$ & $200 \mathrm{~V}$ \\
\hline Nominal current $I_{n}$ & $2000 \mathrm{~A}$ & $2200 \mathrm{~A}$ \\
\hline Current control range & $-2 \mathrm{kA} \ldots+2 \mathrm{kA}$ & $-2.2 \mathrm{kA} \ldots+2.2 \mathrm{kA}$ \\
\hline Ramp rate & $2000 \mathrm{~A} / \mathrm{s}$ & $2200 \mathrm{~A} / \mathrm{s}$ \\
\hline Maximum of power losses & $20 \mathrm{~kW}$ & $25 \mathrm{~kW}$ \\
\hline Current control accuracy & \multicolumn{2}{|c|}{ $\pm 10 \mathrm{~A}$} \\
\hline $\begin{array}{l}\text { Long term stability of } \\
\quad \text { current }(30 \mathrm{~min})\end{array}$ & \multicolumn{2}{|c|}{ $\pm 10 \mathrm{~A}$} \\
\hline $\begin{array}{l}\text { Set minimum current } \\
\text { value } \mathrm{I}_{\min }\end{array}$ & \multicolumn{2}{|c|}{ $\pm 10 \mathrm{~A}$} \\
\hline Residual ripple of current & \multicolumn{2}{|c|}{$5 \mathrm{~A}_{\mathrm{SS}}$} \\
\hline $\cos \varphi$ & \multicolumn{2}{|c|}{$\geq 0.95\left(\right.$ for $\left.\mathrm{I}>75 \% \mathrm{I}_{\mathrm{n}}\right)$} \\
\hline Maximum noise & \multicolumn{2}{|c|}{$70 \mathrm{~dB}(\mathrm{~A})$} \\
\hline EMC & \multicolumn{2}{|c|}{ Class 3} \\
\hline Feedback to the grid & \multicolumn{2}{|c|}{ 12-pulse } \\
\hline
\end{tabular}

In order to have sufficient margin the output parameters were set to $180 \mathrm{~V} / 2 \mathrm{kA}$ for the type A power supplies and to $200 \mathrm{~V} / 2.2 \mathrm{kA}$ for the type B power supply, respectively, even though steady state operation of the coils would only require 110 and $135 \mathrm{~V}$. But the requirement from the physics background for a ramp rate of $2 \mathrm{kA} / \mathrm{s}$ necessitates the higher voltages to force the required rate of change.

The demand for a zero crossing of the current calls for a four quadrant converter as the basic design of the power supply unit. The output feeds from each power supply unit will be three copper cables per pole in parallel with $630 \mathrm{~mm}^{2}$ cross section each. For compensating the relative movement of the coils which are attached to the outer vessel with respect to the cables that are fixed to the torus hall the connection to the coil will be done with highly flexible jumpers connected between the cable and the coil terminals. This will also prevent any undesired forces coming from the high current cables from being transmitted to the coil terminal area. All bolted high current connections will be fitted with MultiContact Seal $\subset$ contact modules to reduce contact resistance and to minimize maintenance effort as the available space at the coil terminal area is very minimal.

\section{B. Cooling system}

For the entire system it is essential that components which show throughout standard operation significant loss of power need to be connected to the IPP's cooling circuit. Therefore a cooling plant with pumps and a heat exchanger has to be designed with respect to the different power supply units cooling circuits. The cooling power will be about $110 \mathrm{~kW}$ in total. To allow a direct cooling of components under voltage a de-ionized water with a conductivity of less than $5 \mu \mathrm{S} / \mathrm{cm}$ will be used.

\section{Control system}

The control system should allow the local control as well as the remote control of the five power supplies from a remote control room, because the power supplies will be located inside of the experimental hall. A superordinated control unit is in charge to handle the remote control of the power supply system by means of a visualization system and the communication with all W7-X main control systems. This includes all connection to the overall operation management, the fast control station and the safety system. The whole control system is based upon Siemens S7C technology including WinCC for visualization. Communication will be established by Profibus DP and Ethernet for all control tasks while the safety systems relies on hardwired connections and a dedicated safety bus.

\section{CONCLUSION}

It has been determined that five trim coils are needed for error field correction. In addition they provide enlarged experimental flexibility for the plasma edge control. Four of these coils are identical in design; the design of the fifth coil had to be different to suit the machine geometry. All coils will be fed individually by independent power supplies.

\section{ACKNOWLEDGMENT}

The authors thank the teams of PPPL and IPP for their effort and the very fruitful co-operation.

\section{REFERENCES}

[1] J. Kißlinger and T. Andreeva, "Correction possibilities of magnetic field errors in Wendelstein 7-X," Fusion Engineering and Design 74 (2005) pp. $623-626$ 